\title{
ALGUNAS REFLEXIONES SOBRE LOS DATIVOS ÉTICOS
}

\author{
JUAN ROMERo \\ Universidad de Extremadura
}

\section{Resumen}

En este trabajo se propone que los dativos éticos son dativos regulares excepto en que al ser adjuntos su derivación se lleva a cabo postsintácticamente. En primer lugar se describen sus propiedades semánticas y morfosintácticas, con especial referencia a la imposibilidad de doblado. Después se esboza el análisis de las construcciones dativas propuesto en Ormazabal y Romero (2010; 2013b). La idea fundamental es que la derivación de la construcción con doblado es distinta a la derivación sin doblado ya que se obtiene por incorporación del pronombre sobre el morfema aplicativo y, a continuación, sobre el verbo. De este modo, no es posible el doblado, lo que explica el comportamiento morfosintáctico de estos dativos, así como su interpretación.

Palabras clave: Dativos éticos, incorporación, aplicativos, laísmo.

\section{SOME REFLECTIONS ON ETHICAL DATIVES}

\begin{abstract}
In this article I propose that ethical datives are regular datives except that, being adjuncts, their derivation is postsyntactic. First, I describe their semantic and morphosyntactic properties, with special reference to the impossibility of clitic doubling. Then, I outline the analysis of dative constructions proposed in Ormazabal \& Romero (2010; 2013b). The main insight is that the derivation of the doubling construction is different from the derivation without doubling, because it is obtained by incorporation of the pronoun on the applicative morpheme and then on the verb. In this way, doubling is not possible, which explains the morphosyntactic behaviour of these datives, as well as their interpretation.

Keywords: Ethic datives, incorporation, applicatives, laísmo.
\end{abstract}




\section{INTRODUCCIÓN}

Los dativos éticos son una rareza sintáctica. Nadie que estudie las relaciones dativas se siente cómodo cuando tiene que lidiar con estos elementos y es por ello que en la mayor parte de los trabajos simplemente se ignoran. Cuando se aborda su estudio (Joitteau y Rezac, 2008; Bosse et al., 2012) se asume que tienen una representación completamente diferente a la del resto de los dativos y normalmente se les atribuye una sintaxis ad hoc. Consideremos el ejemplo prototípico de dativo ético:

\section{(1) El niño no me come}

Desde un punto de vista semántico, el problema fundamental es que no sabemos cómo interpretarlo. Sabemos que suele aparecer en contextos informales, normalmente familiares, y solemos parafrasearlo con enunciados como el niño no come y eso tiene alguna relación conmigo o me afecta de alguna manera. No es algo demasiado preciso, pero no puede ser de otro modo porque sabemos que su uso no afecta a las condiciones de verdad de las oraciones (Bosse et al., 2012) y, por tanto, que es un elemento extraoracional. Por esta razón, los semantistas no quieren saber nada de los dativos éticos.

Por otro lado, desde un punto de vista morfosintáctico, el problema es que su uso no afecta en absoluto ni a las relaciones sintácticas ni a su expresión morfológica, como se manifiesta, por ejemplo, en el hecho de que es el único dativo que puede aparecer en contextos de Restricción de Persona Caso (Bonet, 1991; véase en la sección 2.2 una descripción de esta restricción). Por esta razón, los gramáticos tampoco quieren saber nada de los dativos éticos.

El propósito de este artículo es mostrar que los dativos éticos son elementos mucho más regulares de lo que se ha supuesto hasta ahora. Para empezar, voy a partir de una observación aparentemente trivial, pero que en realidad es muy significativa: los dativos éticos son dativos. Efectivamente, una de las muchas divisiones que podemos hacer entre las lenguas del mundo, muy similar puesto que se hace en términos de caso a la que separa las lenguas ergativas y las nominativas, es aquella que divide las lenguas entre las que expresan el complemento indirecto por medio de un caso específico, el dativo, y aquellas que lo expresan por medio del caso acusativo. En el primer grupo encontramos, por ejemplo, el español, el alemán o el griego y en el segundo el inglés o las lenguas bantúes. Pronombres con las características que encontramos en (1) sólo aparecen, por lo que yo sé, en las lenguas dativas. Además, lo hacen de manera muy productiva: lo normal a luz de los datos es que si una lengua tiene caso dativo también tenga 
dativos éticos ${ }^{1}$. Por el contrario, no existe un acusativo ético: las lenguas que no tienen caso dativo, tampoco tienen un elemento con las propiedades del dativo ético.

Desde este punto de vista, el dativo ético no puede ser ese bicho raro, ese patito feo del que nos hablan, ya que su presencia es completamente sistemática entre las lenguas dativas. En este trabajo voy a repasar primero algunas de sus propiedades principales (sección 2), como la imposibilidad de aparecer en estructuras de doblado. En la sección 3 resumiré brevemente el análisis de la relación dativa que Javier Ormazabal y Juan Romero han defendido en distintos trabajos (especialmente en Ormazabal y Romero, 2007 y 2010). Es imposible presentar estas propuestas con un mínimo detalle en este trabajo, así que me limitaré a esbozar sus características principales, su derivación y los procesos que deben satisfacerse. El lector interesado puede acudir a esos trabajos y a otros que se mencionan en la bibliografía para conocer los detalles. En la sección 4 se mostrará cómo ese mismo análisis puede aplicarse a los dativos éticos y dar cuenta de su distribución y propiedades.

Antes de entrar en materia y dada la confusión terminológica que rodea a esta categoría de elementos (y a tantas otras), debo aclarar que cuando hable de dativos éticos me referiré exclusivamente a los que Salvador Gutiérrez Ordóñez denomina de este modo en la Gramática Descriptiva de la Lengua Española (Gutiérrez Ordóñez, 1999: 1906).

\section{Propiedades de los dativos éticos}

En esta sección vamos a repasar las principales características morfosintácticas de los dativos éticos. No voy a comentar sus propiedades semánticas: su falta de interpretación argumental y su nula contribución a las condiciones de verdad de la oración. Quien desee información sobre estos asuntos puede acudir a los excelentes trabajos de Bosse et al. (2012) o Michelioudakis y Kapogianni (2013) sobre este tema.

En las tres primeras subsecciones trataremos con las propiedades que se manifiestan de manera más clara en español: la imposibilidad de doblar el pronombre, su compatibilidad con la Restricción de Persona Caso y, finalmente, su falta de restricciones a la hora de combinarse con otros pronombres dativos. En la subsección 4 trataremos otras propiedades que, por la

1 Como sugiere un revisor, el asunto es un poco más complejo. Además de asignar dativo, debe tener un sistema de concordancia pronominal, esto deja fuera tanto a lenguas como el japonés, que carecen de concordancia (Romero, 1999), o como el euskera, que se analiza en el apartado 4. 
naturaleza clítica del dativo ético en español, no se manifiestan en nuestra lengua, pero sí en otras como el alemán o el griego.

\subsection{Los dativos éticos no se doblan}

Esta es una de las características más citadas en relación a estos elementos en español. Consideremos el contraste entre (2) y (3).

(2) a. Me dieron el premio

b. Me dieron el premio a mí

(3) a. Ya me han dado el permiso al niño

b. *Ya me han dado el permiso al niño a mí

Como se ejemplifica en el par de (2), los dativos en español permiten el doblado. El dativo puede aparecer únicamente como un pronombre átono (me) o como una combinación concordante entre un pronombre átono y una frase preposicional encabezada por $a$ ( $a$ mí). Como puede verse en (3), la opción del doblado no es posible con los dativos éticos (3b).

Hay que señalar que el doblado es imposible además con otras clases de dativo (4), pero a diferencia de lo que ocurre con los éticos, los pronombres dativos deben en este caso ser correferenciales con el sujeto (5).

(4) a. *Me arrepenti a mi mismo

b. *Me bebi a mi mismo la botella de un trago

(5) a. ${ }^{*}$ Me arrepentiste

b. ${ }^{*}$ Me bebiste la botella de un trago

En el resto de las lenguas, el dativo ético está limitado igualmente a elementos pronominales, en general sólo de primera o segunda persona (Georgala, 2012).

\subsection{No están sujetos a la Restricción de Persona Caso (RPC)}

Como es bien sabido desde las observaciones seminales de Perlmutter (1971) sobre el francés y el español, las combinaciones entre un clítico acusativo y un clítico dativo están muy restringidas. Únicamente son posibles aquellos grupos clíticos en los que el complemento directo es de tercera persona. Obsérvese el contraste entre (6) y (7).

(6) a. Como me porté mal, le mandé un jamón al director

b. Como me porté mal, se lo mandé al director

(7) a. Como te portaste mal, te mandaron al director

b. ${ }^{*}$ Como te portaste mal, te le mandaron al director

En las oraciones de (6) el complemento directo es de tercera persona inanimado y puede aparecer en forma nominal, un jamón, o clítica, lo. 
Cuando aparece en forma de clítico acusativo se combina sin problemas con un pronombre dativo (6b). Por el contrario, en (7) el complemento directo es de primera persona: yo $(=m e)$ soy el «mandado» al director. A diferencia de lo que ocurría en (6), la combinación del pronombre acusativo (me) y el pronombre dativo (le) en (7b) es agramatical (véase Ormazabal y Romero, 2007 para una descripción detallada y una explicación de esta restricción).

La importancia de la RPC radica en su carácter universal. Como ha sido observado por diferentes autores, la RPC se da en todas las lenguas que tienen un sistema de clíticos o de concordancia con los objetos (Albizu, 1997; Romero, 1999; Ormazabal y Romero, 2007; para una generalización distinta, véase Haspelmath, 2004). Por tanto, el hecho de que, como se ejemplifica en (8), los dativos éticos, a diferencia del resto de los dativos, no estén sujetos a esta restricción es morfosintácticamente muy significativo.

(8) ¡Ay, mi hijo, que te me han herido!

A diferencia de lo que ocurre en (7b), el dativo ético, me, es en esta oración perfectamente compatible con el pronombre de objeto directo, $t e$.

\subsection{Es compatible con otros dativos}

Los dativos pueden expresar un cierto número de relaciones semánticas: meta, benefactivo, posesivo, etc. (9). Sin embargo, cuando varias de estas relaciones aparecen simultáneamente en una misma oración, sólo una de ellas puede manifestarse en forma de clítico (10). En el caso del español siempre es la que representa a la meta, esto es, al complemento indirecto típico, pero en otras lenguas es típicamente el benefactivo el argumento que se expresa con el dativo.

(9) a. Le envió una carta (meta)

b. Le preparó un pastel (benefactivo)

c. Le rompió la nariz (posesivo)

(10) a. Enviaron un mensaje a María para ti

b. Le enviaron un mensaje para ti

c. *Te le enviaron un mensaje

Por el contrario, el dativo ético se puede combinar con cualquiera de los anteriores dativos sin inducir agramaticalidad (11).

(11) a. ¡Ay que ya me le han enviado la carta!

b. Mira cómo me le preparo un pastel para cuando vuelva

c. ¡Animales, que me le han roto la nariz! 


\subsection{Otras propiedades}

En español estas son las principales características morfosintácticas. En otras lenguas en las que el dativo ético no es un pronombre átono, tiene otras propiedades que igualmente subrayan su naturaleza esencialmente defectiva con respecto al resto de los dativos. En alemán, por ejemplo, no pueden acentuarse, coordinarse o negarse (12) ni pueden actuar como antecedentes de una relativa o una apositiva (13) (por razones evidentes resulta prácticamente imposible hacer una traducción y, por tanto, las traducciones sólo pretenden orientar al lector del sentido que tendrían las oraciones de ser gramaticales). Los datos están tomados de Georgala (2012).

(12) a. *Da hat er MIR ihm etwas zugeflüstert und... [Entonces ha él ME le algo murmurado y...] [Entonces ME le murmuró algo...]

b. *Komm mir und dem Papa ja pünktlich nach Hause! [Ven me y el padre PARTCL puntual a casa] [;Ven por mi y por tu padre a casa puntualmente!]

c. *Das war nicht dir, sondern mir ein Spaß! [Esto era no te, sino me una diversión] [Esto no me sino te era divertido]

(13) a. *Komm mir, die ich mich um dich Sorge, pünktlich nach Hause! [Ven me que yo mismo por tu cuidado puntual a casa] [Venme puntual a casa, que te cuidaré]

b. *Der war dir, seiner besorgten Mutter, betrunken! [Él era te, POSS.3SG.MASC.DAT preocupada madre, bebido]

Efectos similares a los descritos para el alemán y otros se dan en lenguas como el griego o el francés (Georgala, 2012; Joitteau y Rezac, 2008).

\subsection{Conclusión}

En suma, por un lado hemos visto que los dativos éticos aparecen única, pero productivamente, en las lenguas que tienen caso dativo y que no existe nada semejante en las lenguas que carecen de este caso. Sin embargo, también hemos visto que la morfosintaxis del caso dativo es en muchos aspectos completamente distinta a la del resto de dativos. Así pues, el problema salta a la vista: ¿por qué algo que es típicamente dativo tiene un comportamiento tan poco dativo?

En la sección 4 propondremos un análisis para este tipo de dativos basado en la idea de que los dativos éticos son estructuralmente idénticos a los dativos sin doblar. El análisis de los dativos lo presentamos en la sección 3 y esboza las propuestas de Ormazabal y Romero (2007, 2010 y 2013b). Propondremos que, dado su estatus no argumental, no entran en operaciones de legitimación o cotejo con el verbo y, por este motivo, no entran en conflicto con otros argumentos. 


\section{LA ESTRUCTURA DE LA RELACión DATIVA}

Voy a asumir un análisis derivacional de la relación dativa por el que (14b) se deriva de (14a) (Larson, 1988; Baker, 1988; Ormazabal y Romero, 2010):

(14) a. Puso las patas en la mesa

b. Le puso las patas a la mesa

La derivación se representa de manera gráfica en (15):

(15) a.

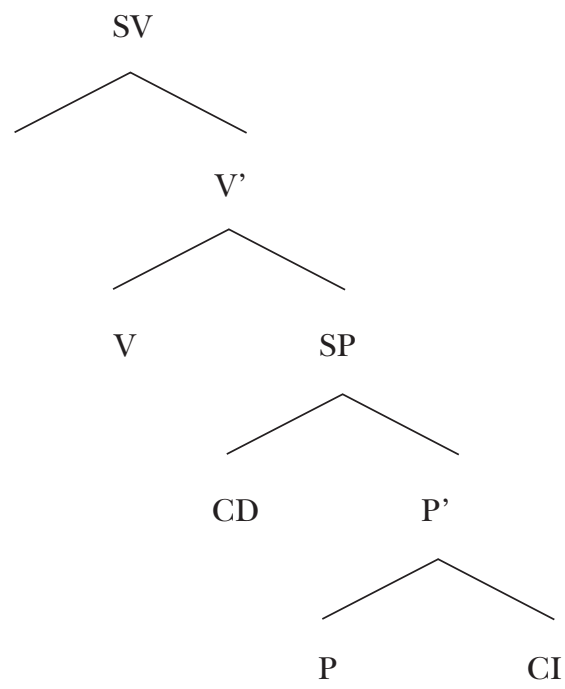

b.

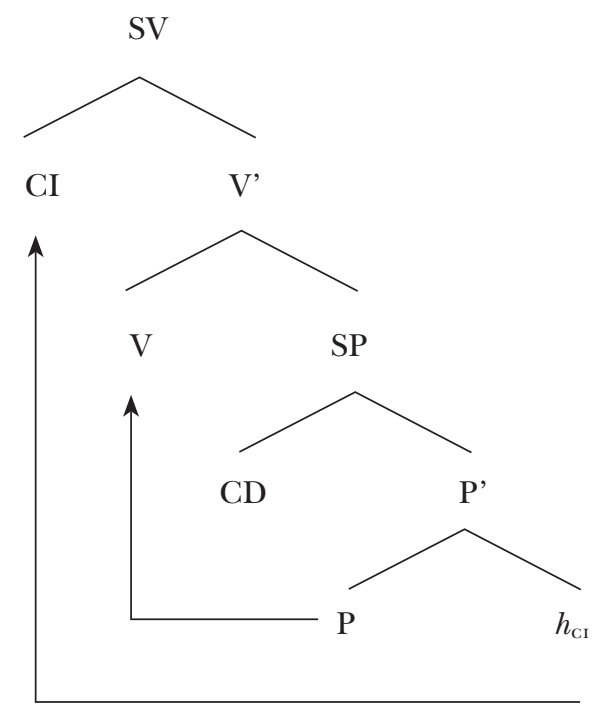

AEF, vol. XLII, 2019, 233-245 
El proceso para formar la relación dativa consta de dos relaciones sintácticas. En la primera se establece una relación entre el verbo y la preposición. Esencialmente es un reflejo de la relación de selección semántica por la que los verbos ditransitivos seleccionan una meta en un esquema actancial del tipo (X causa [que Y vaya a Z]) (enviar, decir, etc.). El reflejo morfosintáctico de esta relación es la incorporación de la preposición sobre el verbo. Este proceso puede verse de manera transparente en muchas lenguas habitualmente bajo el nombre de morfema aplicativo, por ello no insistiré más en este asunto (véase Peterson, 2007).

La segunda relación consiste en la asignación de caso al CI, en español Marcado Diferencial de Objeto (MDo). Se trata exactamente de la misma relación que se establece entre el verbo y los llamados objetos directos preposicionales (Pensado, 1995) ${ }^{2}$. Obsérvese el contraste entre las oraciones (16-17):

(16) a. Pusieron un vigilante en el edificio

b. Le pusieron un vigilante al edificio

(17) a. [Como vigilante] pusieron a su hermano en el edificio

b. *[Como vigilante] le pusieron a su hermano al edificio

En (16) vemos que la construcción «poner un vigilante» es compatible con una relación dativa (16b). Sin embargo, cuando el objeto directo requiere mdo (17), la relación de dativo se bloquea (véase Ormazabal y Romero, 2013a para un análisis detallado de este bloqueo). En consecuencia, parece plausible asumir que el dativo y el complemento directo compiten por una misma relación de Caso: el moo.

Consideremos ahora una oración como (18):

(18) [Al director] le mandaron a la alumna discola al despacho

Lo interesante de (18) es que, en contra de lo que acabamos de ver, el complemento directo, a la alumna díscola, puede recibir MDo. En Ormazabal y Romero (2013b) y Romero (en prensa) se propone una explicación a este hecho basada en que la derivación propuesta en (15) no es la única posibilidad que ofrece el componente computacional. No hay nada en el sistema, tal y como está definido, que pueda evitar que el complemento de $\mathrm{P}$ en (15) se incorpore sobre $\mathrm{P}$ y a continuación sobre V (19) si el resultado de esa operación puede representarse morfológicamente. De este modo, el complemento indirecto no recibe MDo, ya que los elementos incorporados no establecen relaciones ni de caso ni de concordancia (Baker, 1988). De este modo, el verbo puede asignar mDo al complemento directo, del mismo

2 Dado que se trata de la misma relación, me referiré a ella de manera general como Marcado Diferencial de Objeto, siguiendo la terminología de Georg Bossong. 
modo que en una oración pasiva se puede asignar caso nominativo al complemento directo de la oración activa correspondiente ${ }^{3}$.

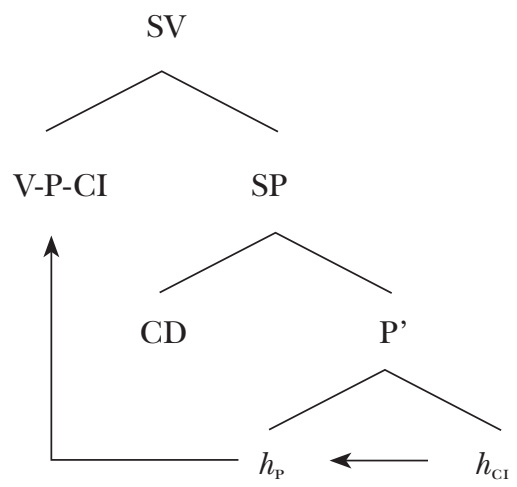

Nótese que el análisis de (19) no sólo es perfectamente compatible con un modelo derivacional, sino que es precisamente lo que esperamos. En los modelos derivacionales no se pueden imponer condiciones para obtener una cierta construcción, ya que las construcciones en sí no son más que meros artefactos descriptivos. Por el contrario, esperamos que todas las posibilidades derivacionales aparezcan siempre que sea posible expresarlas morfológicamente. En otras palabras, las derivaciones de (15) y (19) son dos derivaciones distintas que se expresan por medio de los mismos clíticos.

Ahora bien, dado que se trata de derivaciones distintas, es razonable esperar que en algún dialecto se manifieste esa diferencia. De hecho, una prueba en favor de esta propuesta es que en los dialectos laístas las dos derivaciones difieren en su expresión morfológica:

(20) a. Le di un regalo a María

b. La di un regalo

En el dialecto laísta que se habla comúnmente en Madrid (Quilis et al., 1985) el uso de la está generalizado cuando no hay doblado (20b), pero está muy restringido cuando sí lo hay (20a) (véase Romero, 2013 para más contrastes en este mismo sentido).

En suma, la relación dativa se puede obtener en español con dos derivaciones distintas, (15b) y (19), a partir de una misma representación en la base (15a). En la siguiente sección propondremos que esta misma derivación puede dar cuenta de las propiedades de los dativos éticos.

${ }^{3}$ De hecho, el análisis de Larson (1988) establece explícitamente una relación entre la derivación de las oraciones pasivas y de las oraciones aplicativas. 


\section{Los DAtivos Éticos}

Todas las derivaciones que se han presentado en la sección anterior tienen lugar en el componente sintáctico, como se muestra claramente en el hecho de que están sujetas a la RPC. La propuesta para derivar los dativos éticos es que se trata esencialmente de la misma derivación que representamos en (19), pero en Forma Fonética. De este modo se predice que el dativo ético no interferirá con ningún proceso sintáctico y, al mismo tiempo, se explica su estatus dativo.

Antes de entrar en el análisis es preciso hacer una puntualización. Los dativos éticos forman parte de un conjunto de elementos más amplio y de naturaleza semánticamente extraproposicional (parentéticos, vocativos, etc.). Tanto en las lenguas dativas como en las no dativas es posible encontrar, por ejemplo, un tipo de morfemas llamados alocutivos, que comparten algunas propiedades con los dativos éticos. Considérense, por ejemplo, los siguientes ejemplos de kinyarwanda (21) y euskera (22) (Ngoboka, 2005; Moreno Cabrera, 1998):

(21) Umukoobwa a-ku-nyw-eer-a inzoga [mujer 1sS-2sO-beber-app-fo cerveza] [La mujer te está bebiendo cerveza]

(22) a. Gizon bat etorri $d u-k$ [Hombre uno venido ha-te.Masc] [Te ha venido un hombre (hablando con un hombre)]

b. Gizon bat etorri $d u-n$ [Hombre uno venido ha-te.Fem] [Te ha venido un hombre (hablando con una mujer)]

Voy a dejar estos casos fuera porque aunque en algunos sentidos tienen propiedades semejantes a las de los dativos éticos (en kinyarwanda, por ejemplo, aparece el mismo morfema aplicativo, app, que con los complementos indirectos), se trata de elementos que sólo pueden aparecer en segunda persona y que son obligatorios en ciertos contextos (Eguren, 2000). Esto, añadido al hecho de que no son necesariamente dativos, hace que tengan características morfosintácticas propias que deben recibir un análisis específico. No obstante, no puede descartarse que algunos de estos morfemas alocutivos puedan recibir estructuralmente el mismo análisis que los dativos éticos.

En las lenguas no dativas es posible encontrar también elementos similares al dativo ético pero en forma de Sintagma Preposicional:

(23) The cat died on me

[El gato murió en mí]

[El gato se me murió] 
Nótese que este tipo de elementos pueden representarse directamente por medio de la estructura propuesta en (15a): la preposición on ocupa el núcleo del SP y me la posición de complemento de P. Así pues, la pregunta que debemos hacernos es por qué no pueden derivarse de la forma en la que se derivan normalmente este tipo estructuras en las lenguas no dativas. Esto es, por qué no podemos derivar algo similar a lo que vemos en (24b), la llamada Construcción de Doble Objeto.

(24) a. I sent a book to Mary [Yo envié un libro a Mary]

b. I sent Mary a book [Yo le envié un libro a Mary]

En Ormazabal y Romero (2007) se argumenta que la diferencia entre una clase de lenguas y la otra radica precisamente en las propiedades de P. En español y en las lenguas dativas, P establece una relación formal con su complemento independiente de la que establece V. Esto explica por qué en las lenguas dativas la pasivización siempre se hace con el complemento directo (25a), mientras que en las lenguas no dativas se pasiviza el complemento indirecto $(25 \mathrm{~b})^{4}$.

(25) a. Un libro le fue enviado a María (frente a *María fue enviada un libro) b. Mary was sent a book (frente a *A book was sent Mary)

Así pues, la clave de la diferencia entre una y otra clase de lenguas radica en las propiedades formales de P. Hemos visto que hay dos derivaciones posibles. En la primera (15b) (i) la preposición se incorpora y (ii) se establece una relación de Caso y concordancia entre $[\mathrm{V}+\mathrm{P}]$ y el complemento indirecto. En la segunda (19), el complemento indirecto se incorpora sobre $\mathrm{P}$, por lo que ya no necesita Caso (Baker, 1988), y P se incorpora sobre V para cotejar sus rasgos de concordancia/animacidad (Ormazabal y Romero, 2007; Richards, 2008).

Como ya se ha dicho, el dativo ético no tiene ninguna relación semántica con el verbo. Por tanto, podemos asumir que en este caso el complemento indirecto se incorpora sobre $\mathrm{P}$, pero al no cotejar ningún rasgo del verbo, $\mathrm{P}$ no se incorpora sobre V, sino que simplemente se adjunta a V en Forma Fonética por razones meramente prosódicas: necesita acento. De este modo podemos dar cuenta de todas las propiedades descritas.

4 De hecho, en las lenguas en las que las preposiciones pueden expresar concordancia, como las lenguas celtas, no existe la alternancia ditransitiva ejemplificada en (16) o en (24). Así pues, parece que la relación dativa es una solución de compromiso cuando P posee una serie de rasgos formales (de caso y concordancia), pero no puede expresarlos morfológicamente. 
En primer lugar, no se pueden doblar porque están incorporados y, por tanto, siguen una derivación similar a la que describimos en (19). En segundo lugar, no interfieren con otras relaciones porque no se desplazan sobre el verbo en el componente sintáctico, sino en $\mathrm{FF}$, quizá por su estatus de adjunto (Stepanov 2001). Finalmente, por este mismo motivo son compatibles con otros dativos.

Quedan aspectos por explicar, desde luego, por ejemplo, por qué debe incorporarse el pronombre y no aparecer como en inglés en forma de SP. Es posible que esto tenga que ver con su naturaleza expresiva que normalmente limita su uso a la primera y segunda persona y, por tanto, pueda derivarse de las propiedades generales de los pronombres en español. Sin embargo, creo que este trabajo ofrece una vía muy prometedora, que no requiere de ninguna maquinaria que no esté independientemente justificada, para responder a la pregunta que planteábamos anteriormente: ¿por qué unos elementos tan típicamente dativos tienen un comportamiento tan poco dativo? Así mismo, en la medida en que este análisis se encuentre en la buena dirección supone una evidencia adicional para los modelos derivacionales para los que las construcciones sintácticas no son más que un mero artefacto descriptivo sin valor conceptual.

\section{Bibliografía}

Albizu, P. (1997): «Generalized PCC». En Mendikoetxea, A. y Uribe-Etxebarria, M. (eds): Theoretical issues on the morphology-syntax interface. Donosti, Universidad del País Vasco, págs. 1-33.

BAKer, M. (1988): Incorporation. Chicago, University of Chicago Press.

Bonet, E. (1991): Morphology after Syntax. Tesis doctoral. Massachusetts Institute of Technology.

Bosse, S. et al. (2012): «Affected Experiencers». Natural Language and Linguistic Theory 30.4, págs. 1185-1230.

Eguren, L. (2000): «El morfema de alocutivo del euskera y el modelo de gramática». Hermeneus, 2, págs. 95-118.

Georgala, E. (2012): Applicatives in their structural and thematic function: A minimalist account of multitransitivity. Tesis doctoral. Cornell University.

Gutiérrez Ordóñez, S. (1999): «Los dativos». En Bosque, I. y Demonte, V. (eds): Gramática descriptiva de la lengua española. Madrid, Espasa, vol. 2, págs. 1855-1930.

Haspelmath, M. (2004): «Explaining the ditransitive person-role constraint: A usagebased approach». Constructions, 2 (en línea: <http://www.constructions-online.de, urn:nbn:de:0009-4-359> [consulta: 22 de junio de 2018]).

Jouitteau, M. y Rezac, M. (2008): «The French ethical dative, 13 syntactic tests». Bucharest Working Papers in Linguistics, 9.1, págs. 97-108. 
Larson, R. (1988): «On the Double Object Construction». Linguistic Inquiry, 19, págs. 335-391.

Michelioudakis, D. y Kapogianni, E. (2013): «Ethical datives. A puzzle for syntax, semantics, pragmatics, and their interfaces». En Folli, R. et al. (eds): Syntax and its Limits. Oxford, Oxford University Press, págs. 345-369.

Moreno Cabrera, J.C. (1998): «Allocutivity and voice in the Basque verb». En Kulikov, L. y Vater, H. (eds): Typology of verbal categories. Tübingen, Max Niemeyer Verlag, págs. 169-178.

Ngoboka, J.P. (2005): A syntactic analysis of Kinyarwanda applicatives. Tesis doctoral. Universidad de KwaZulu-Natal.

Ormazabal, J. y Romero, J. (2007): «The Object Agreement Constraint». Natural Language and Linguistic Theory, 25.2, págs. 315-347.

- (2010): «The derivation of dative alternations». En Duguine, M. et al. (eds.): Argument Structure and Syntactic Relations from a Crosslinguistic Perspective. AmsterdamPhiladelphia, John Benjamis, págs. 203-232.

- (2013a): «Object Agreement, Clitics and Dialectal Variation». Probus, 25, págs. 301-344.

- (2013b): «Non accusative objects». Catalan Journal on Linguistics, 12, págs. 155-173.

Pensado, C. (ed.) (1995): El complemento directo preposicional. Madrid, Visor Libros.

Perlmutter, D. (1971): Deep and Surface Structure Constraints in Syntax. New York, Holt, Rinehart and Winston, Inc.

Peterson, D.A. (2007): Applicative constructions. Oxford, Oxford University Press.

Quilis, A. et al. (1985): Los pronombres le, la, lo y sus plurales en la lengua española hablada en Madrid. Madrid, CSIC.

Richards, M. (2008): «Defective agree, case alternations, and the prominence of person». En Richards, M. y Malchukov, A.L. (eds.): Scales. Leipzig, Universität Leipzig (Linguistische Arbeits Berichte, 86).

Romero, J. (1999): «The case of agreement». En Arregui, K. et al. (eds.): Papers on syntax and morphology. MITWPL, 33, págs. 343-368.

- (2013): «Accusative datives in Spanish». En Fernández, B. y Etxepare, R. (eds.): Variation in datives: a microcomparative perspective. Oxford, Oxford University Press, págs. 283-300.

- (en prensa): «Empty categories and clitics». En Gallego, A. (ed.): The Syntactic Variation of Spanish Dialects. Oxford, Oxford University Press.

Stepanov, A. (2001): «Late adjunction and Minimalist Phrase Structure». Syntax, 4.2, págs. 94-125. 